\title{
Modified autotransplantation technique for surgical resection of complex pericardial synovial sarcoma
}

\author{
Klaudiusz Stoklosa ${ }^{1}$, Nazari Dvirnik ${ }^{2}$, and Robert Cusimano ${ }^{2}$ \\ ${ }^{1}$ University of Toronto Temerty Faculty of Medicine \\ ${ }^{2}$ Toronto General Hospital
}

January 1, 2022

\begin{abstract}
Technical details for complex cardiac tumor resection are sparse. We describe the operative technique of modified autotransplantation for resection of a complex pericardial synovial sarcoma in a 63-year-old, Caucasian female. Surgical exposure demonstrated tumor origin at the superior cavoatrial junction and invasion of the aorta, main pulmonary artery, superior pulmonary veins, and left atrial roof. Full macroscopic surgical resection was achieved. The patient received adjuvant radiation for microscopic positive margins and remains alive and with no tumor progression at one year postoperatively. We conclude that modified autotransplantation is a challenging but effective surgical technique when performed with careful patient selection and availability of skilled, cardiothoracic surgeons at a cardiac center of excellence.
\end{abstract}

\section{INTRODUCTION}

Pericardial synovial sarcoma is one of the rarest primary cardiac tumors, with a median overall survival of 27 months. ${ }^{1}$ Surgical resection is the main factor associated with prolonged survival. ${ }^{2}$ Unfortunately operative guidelines have not been standardized given the rarity, heterogeneity, and anatomical complexity, including intramyocardial involvement and left-sided tumors.

Autotransplantation has been shown to be a feasible technique for complex, left-sided cardiac tumor resection. ${ }^{3}$ While rarely used for cardiac tumors, partial autotransplantation techniques offer left atrial access with decreased ischemic time. ${ }^{4-7}$ We present the second reported case of pericardial synovial sarcoma treated with modified autotransplantation and adjuvant radiation.

\section{CASE PRESENTATION}

An incidental finding of cardiomegaly was found on the chest x-ray of a 63-year-old Caucasian female. Follow-up out-patient CT scan revealed a heterogeneous $108 \times 57 \times 89$-mm pericardial lesion encasing the heart. Histopathologic biopsy identified spindle cell, pericardial synovial sarcoma with SS18-SSX1 fusion gene, positive for bcl2 and CD99. No metastases were found on radiographic imaging. She had a negative cardiac review of symptoms and echocardiography suggested no significant pericardial tamponade or effusion at initial diagnosis. Her family history was negative for cardiac concerns.

The patient completed six cycles of neoadjuvant doxorubicin and ifosfamide with tumor response. She developed hemorrhagic cystitis, febrile neutropenia, and significant anemia with her last chemotherapy cycle requiring an ICU admission. Post-chemotherapy cardiac MRI (Figure 1, $A$ - $B$ ) suggested tumor fixation to the superior cavoatrial junction, encasing but likely not invading the great vessels and superior pulmonary veins, with potential for invasion of the roof of the left atrium. A 3D model of the tumor was undertaken using the Sketchfab platform (www.sketchfab.com) (Figure 1,C-D ) to provide better pre-operative anatomic 
and thus surgical understanding. After multidisciplinary discussion and treatment, the patient was offered operative intervention following completion of her chemotherapy.

\section{OPERATIVE TECHNIQUE}

A team of highly specialized cardiothoracic surgeons used a modified cardiac autotransplantation technique for tumor resection. Standard midline sternotomy was used. The patient was placed on cardiopulmonary bypass after arterial cannulation of the mid-aortic arch and bicaval cannulation, being careful to cannulate as superior as possible in the SVC. Tumor dissection began while on cardiopulmonary bypass prior to aortic cross-clamp. Antegrade cardioplegia was administered every 20 minutes.

The modified autotransplantation approach was performed by transecting the SVC, aorta and main pulmonary artery, leaving the IVC in situ. Following this, the left and right atria were entered allowing for tumor resection. The tumor had invaded the adventitia of the main pulmonary artery. The superior half of the right atrium and inferior half of the superior vena cava (SVC), inferior to the azygous vein, were resected en bloc with the tumor as was the roof of the left atrium, main pulmonary artery, circumflex coronary artery and portions of the aortic root (Figure 2, $A-B$ ). Both the left and right superior pulmonary vein-left atrial junctions were resected with the specimen. Complete macroscopic tumor resection was accomplished.

With the heart attached by the inferior pulmonary veins and IVC, it was raised such that the left atrium was anterior. Bovine pericardium was used to reconstruct the anterior and superior aspects of the left atrium. The superior pulmonary veins were reconstructed and re-anastomosed to the left atrium. The main pulmonary artery, ascending aorta, aortic root, and right atrium were reconstructed using bovine pericardium in this sequence. A piece of great saphenous vein was then anastomosed in end-to-end fashion from the left circumflex artery in the atrioventricular groove to the reconstructed noncoronary sinus of the ascending aorta. Total cross-clamp, pump, and re-perfusion times were 251 minutes, 383 minutes, and 72 minutes, respectively. Severe coagulopathy was managed with seven units of platelets, five units of plasma, 16 units of blood, and prothrombin complex. An epicardial dual-chamber pacemaker was also implanted at the operation due to SA node resection.

The resected tumor was measured as 110 x 60 x 57-mm and weighed $293.8 \mathrm{~g}$. Pathology report confirmed the diagnosis of spindle cell, pericardial synovial sarcoma with 8 mitoses per 10 high power fields, $80 \%$ viable tumor, and no necrosis. The tumor was staged as ypT1. Despite best surgical efforts, some frozen sections (aorta and left atrial roof) had microscopic positive margins.

\section{POSTOPERATIVE STATUS}

The patient's postoperative course included thrombocytopenia, with a corresponding negative heparininduced thrombocytopenia assay, and atrial fibrillation managed with amiodarone. She was started on warfarin with a target INR of 2.0-3.0. Postoperative echocardiography demonstrated left ventricular ejection fraction of $45-50 \%$, right ventricular volume and pressure overload, moderately reduced right ventricular systolic function, and mild mitral and tricuspid regurgitation. She was discharged home on the $14^{\text {th }}$ postoperative day.

The patient has been recovering well. She has completed a postoperative, five-week course of adjuvant external radiation for the microscopic positive margins, delivered as 60 Gy in 25 fractions. Follow-up chest, abdomen, and pelvis CT have suggested no new or progressive disease in the chest or metastases. She is alive and free from tumor recurrence at one year postoperatively.

\section{DISCUSSION}

Primary pericardial synovial sarcoma is an exceedingly uncommon, highly aggressive neoplasm. Despite the striking predominance for males between adolescence and early youth, we present a 63 -year-old Caucasian female with extensive tumor superior to the fibrous cardiac skeleton. ${ }^{1,8}$

Due to its extreme rarity, most knowledge about this sarcoma, including surgical management, is limited to case reports. Many surgical nuances are omitted thereby leaving surgeons with little guidance for details 
regarding operative resection and reconstruction. Incomplete resections have previously been described due to tumor complexity and extent of invasion. ${ }^{9,10}$ In particular, Yano et al. describe an 18-year-old male patient with a pericardial synovial sarcoma in nearly the exact anatomical pattern as our patient's presentation. However, Yano et al. explain that complete tumor resection was not feasible at the time due to the extent of invasion. One must wonder whether guidance on the modified autotransplantation technique and subsequent adjuvant treatments would have proved beneficial in preventing their patient's death from local recurrence.

The modified autotransplantation approach offers a great alternative to the full autotransplantation technique described in cardiac cancer literature. ${ }^{3}$ Full resection of the heart is not always required and complicates reconstruction, with the potential to cause problems, particularly if the surgeon is not familiar with cardiac transplantation. Even with transplantation experience, complications from IVC reconstruction are possible, especially from ostial coronary sinus distortion or stenosis which can lead to death. ${ }^{11}$ Not disrupting the IVC also eliminates the risk of IVC stenosis and obstruction, which have been previously described with the bicaval orthotopic heart transplant technique and caused by edema at the suture lines, thrombus, and tight anastomoses. ${ }^{12-14}$ At times, IVC stenosis and obstruction may be less clinically obvious, with reports of diagnosis up to two months postoperatively. ${ }^{12,15}$ However, there is a risk of profound hemodynamic compromise, hepatic failure, or multi-organ systemic failure if this rare but catastrophic complication is not immediately corrected. ${ }^{13}$ Furthermore, time saved on reconstruction will add to the overall benefit of the operation. Modified autotransplantation allows for significant tumor resection with less reconstruction, re-implantation, and ischemic time on these very complex and lengthy procedures compared to a full explant. It facilitates the principle of maximal tumor resection with minimal surgical manipulation. It is for these reasons that full autotransplantation is rarely undertaken.

There are many intricacies to the modified autotransplantation approach. SVC cannulation for cardiopulmonary bypass should be undertaken carefully and be placed as superior as possible to maintain enough cava for re-anastomosis to the reconstructed heart. Moreover, incision into the SVC should be oblique to decrease the likelihood of subsequent stenosis. Transection of the superior great vessels leads to wonderful exposure of the great vessels and base of the heart. It is the first step in considering what else must be transected to allow for adequate tumor extirpation without compromising usual cardiac attachments and their potential detrimental consequences. Reconstruction of the origin of the pulmonary veins may be fraught with danger; however, downstream complications may be minimized if late pulmonary vein stenosis can be avoided. One must realize that bovine pericardium is inelastic and may be prone to stenosis either by scar or by undersizing neo-conduits or openings into a flaccid and thus contracted pulmonary vein opening. This complication must be anticipated at the initial reconstruction as it often takes time to manifest. Furthermore, cardiac distortion occurs with simultaneous right-left atrial reconstruction. Therefore distortion related to mal-reattachment of the reconstructed heart to the body is also a danger, particularly if usual landmarks for re-connection may be missing or misunderstood. While none of these are insurmountable, any reduction in potential complications related to re-attachment of the resected and reconstructed heart is desirable.

It is noteworthy that loss of atrial contractility is often fairly well tolerated even with complete atrial reconstruction. ${ }^{2}$ Patients may regain cardiovascular function to a tolerable degree. However, this has not been studied clearly. We have seen patients at our institution demonstrating signs and symptoms of cardiac failure despite reasonable ventricular function following complete atrial resection. It is unclear whether this is a consequence of atrial noncompliance or a lack of an atrial kick. We have seen evidence of otherwise unexplainable pulmonary hypertension and peripheral edema in patients with variously reconstructed atria, including partial reconstruction. For this reason, atrial reconstruction should be performed while leaving large residual atria. It is important to note that extensive ventricular tumor involvement will limit the surgical resection and such patients may not be the best candidates for an autotransplantation. ${ }^{2}$

Autotransplantation, either complete or modified, is highly dependent on surgical experience and skillset. The adage "only remove as much as YOU can reconstruct" is key. Extensive experience in cardiac transplantation and availability of skilled cardiothoracic surgeons are necessary for safe execution. Notably, the only other case report of a modified autotransplantation technique for a primary synovial sarcoma was performed 
by the same surgeon and center as in this case report. ${ }^{7}$

\section{CONCLUSION}

Primary pericardial synovial sarcomas are exceedingly uncommon. Complete surgical resection is critical to prolong long-term survival. Technical surgical guidance for complex tumors is lacking. This report describes the successful resection of an extensive and invasive pericardial synovial sarcoma using a modified autotransplantation technique with adjuvant radiation for microscopic positive margins. Surgical technical aspects are described. Our case can be of benefit to cardiac surgeons embracing challenging cases. Overall, careful patient selection, a thorough surgical plan and understanding of anatomy and physiology, expertise in transplantation, and availability of skilled, senior cardiothoracic surgeons at a center of excellence are critical for success. The modification of resection as required may help improve outcomes.

\section{AUTHOR CONTRIBUTIONS}

All authors were involved in data acquisition, manuscript drafting and revision, and approval of final version for submission. Dr. Cusimano was the primary surgeon performing this modified autotransplantation for tumor resection.

\section{REFERENCES}

${ }^{1}$ Duran-Moreno J, Kampoli K, Kapetanakis EI et al. Pericardial Synovial Sarcoma: Case Report, Literature Review and Pooled Analysis. In vivo 2019;33:1531-1538.

2 Cusimano RJ. Surgical Management of Cardiac Tumors. Semin Diagn Pathol 2008;25:76-81.

${ }^{3}$ Reardon MJ, Malaisrie SC, Walkes JC et al. Cardiac Autotransplantation for Primary Cardiac Tumors. Ann Thorac Surg 2006;82:645-650.

${ }^{4}$ Erdogan HB, Kirali K, Omeroglu SN et al. Partial Cardiac Autotransplantation for Reduction of the Left Atrium. Asian Cardiovasc Thorac Ann 2004;12:111-114.

${ }^{5}$ Pan J, Li QG, Li J, Wang DJ. Partial cardiac autotransplantation with a concomitant mitral valve, aortic valve replacement and tricuspid plasty. ICVTS 2013;17(5):906-907.

${ }^{6}$ Lessana A, Scorsin M, Scheuble C et al. Effective Reduction of a Giant Left Atrium by Partial Autotransplantation. Ann Thorac Surg 1999;67 (4):1164-1165.

7 Menegazzo WR, Alvarez J, Cusimano RJ et al. Modified autotransplant with three-dimensional printing for treatment of primary cardiac sarcoma. JTCVS 2018;157(2):e41-43.

8 Coli A, Chiariello GA, Novello M et al. Treatment of cardiac synovial sarcoma: experience of two cases. J Cardiothorac Surg 2018;13(84).

9 Yano M, Toyooka S, Tsukuda K et al. SYT-SSX fusion genes in synovial sarcoma of the thorax. Lun Cancer 2004;44(3):391-397.

${ }^{10}$ Yoshino M, Sekine Y, Koh E et al. Pericardial synovial sarcoma: a case report and review of the literature. Surg Today 2014;44(11):2167-2173.

11 Oniscu GC, Forsythe HLR, Pomfret EA. Transplantation Surgery: Orthotopic Heart Trasnplantation. Springer. 2019;2:35-60.

12 Bleasdale RA, Partridge J, Banner NR. Obstruction of the inferior vena cava following total heart lung transplantation: successful treatment by balloon angioplasty. J Heart Lung Transplant 2000;19:488-491.

13 Abudan A, Kidd B, Hild P, Gupta B. Obstruction of the inferior vena cava following bicaval orthotopic heart transplantation: a case series. Eur Heart J Case Rep. 2021;5(2):ytab046.

14 John R, Liao K. Orthotopic heart transplantation. Oper Tech Thorac Cardiovasc Surg. Elsevier Inc. 2010; 15:138-146. 
15 Jacobsohn E, Avidan MS, Hantler CB, et al. Case report: Inferior vena-cava right atrial anastomotic stenosis after bicaval orthotopic heart transplantation. Can J Anesth. 2006;53(10):1039-1043.

\section{FIGURES}

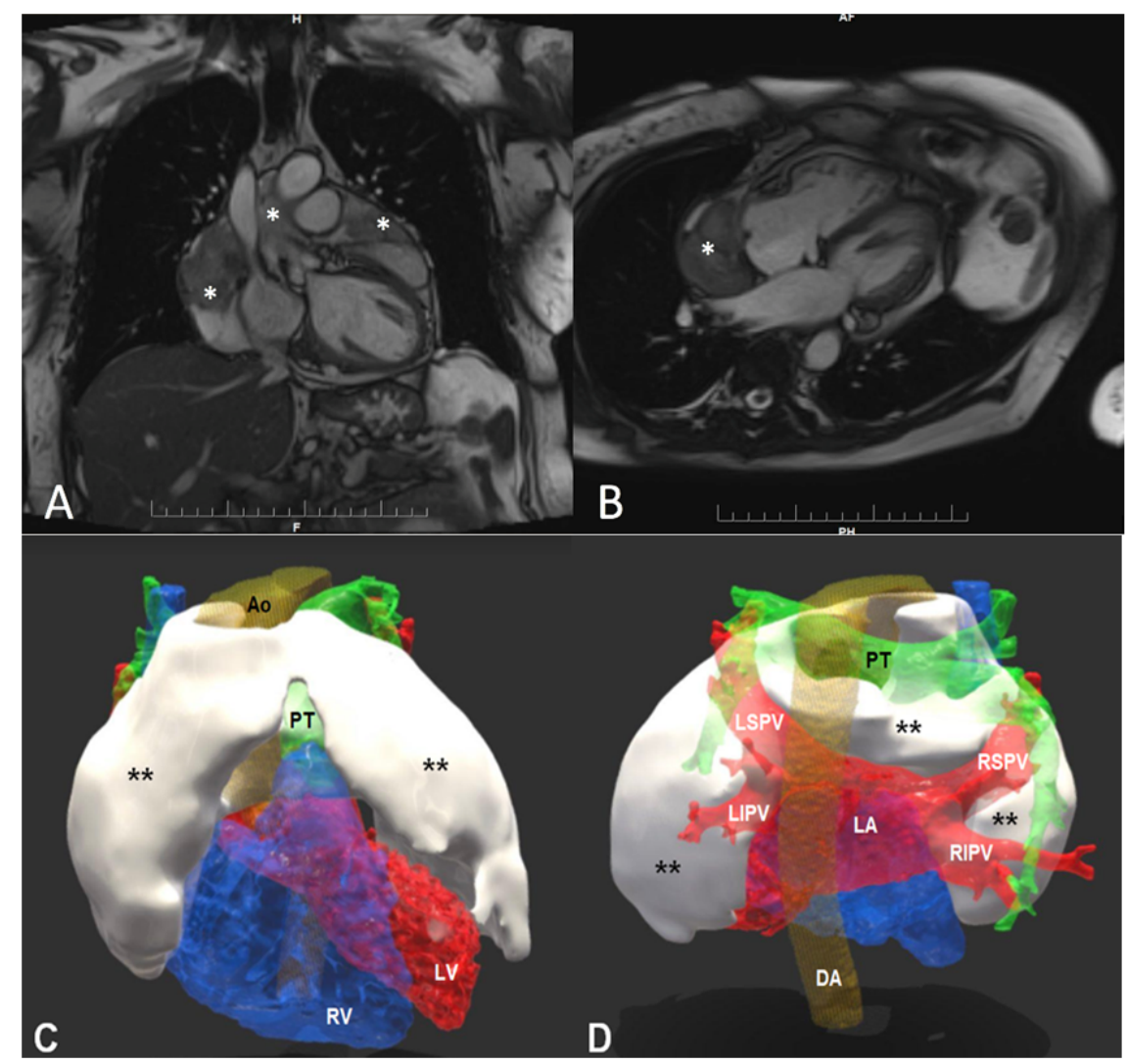

FIGURE 1. Pre-operative tumor visualization. A and B, (A) Coronal and (B) axial post-chemotherapy cardiac MRI, demonstrating the location of the tumor at the lateral borders of the heart (asterisk). C and $\mathrm{D},(\mathrm{C})$ anterior and (D) posterior view of the reconstructed three-dimensional tumor (white, double asterisk ) encasing the great vessels. H: Head. F: Foot. AF: Anterior. PH: Posterior. Ao: Ascending aorta. DA: Descending aorta. LA: Left atrium. LV: Left ventricle. LIPV: Left inferior pulmonary vein. LSPV: Left superior pulmonary vein. RIPV: Right inferior pulmonary vein. RSPV: Right superior pulmonary vein. RV: Right ventricle. PT: Pulmonary trunk. 


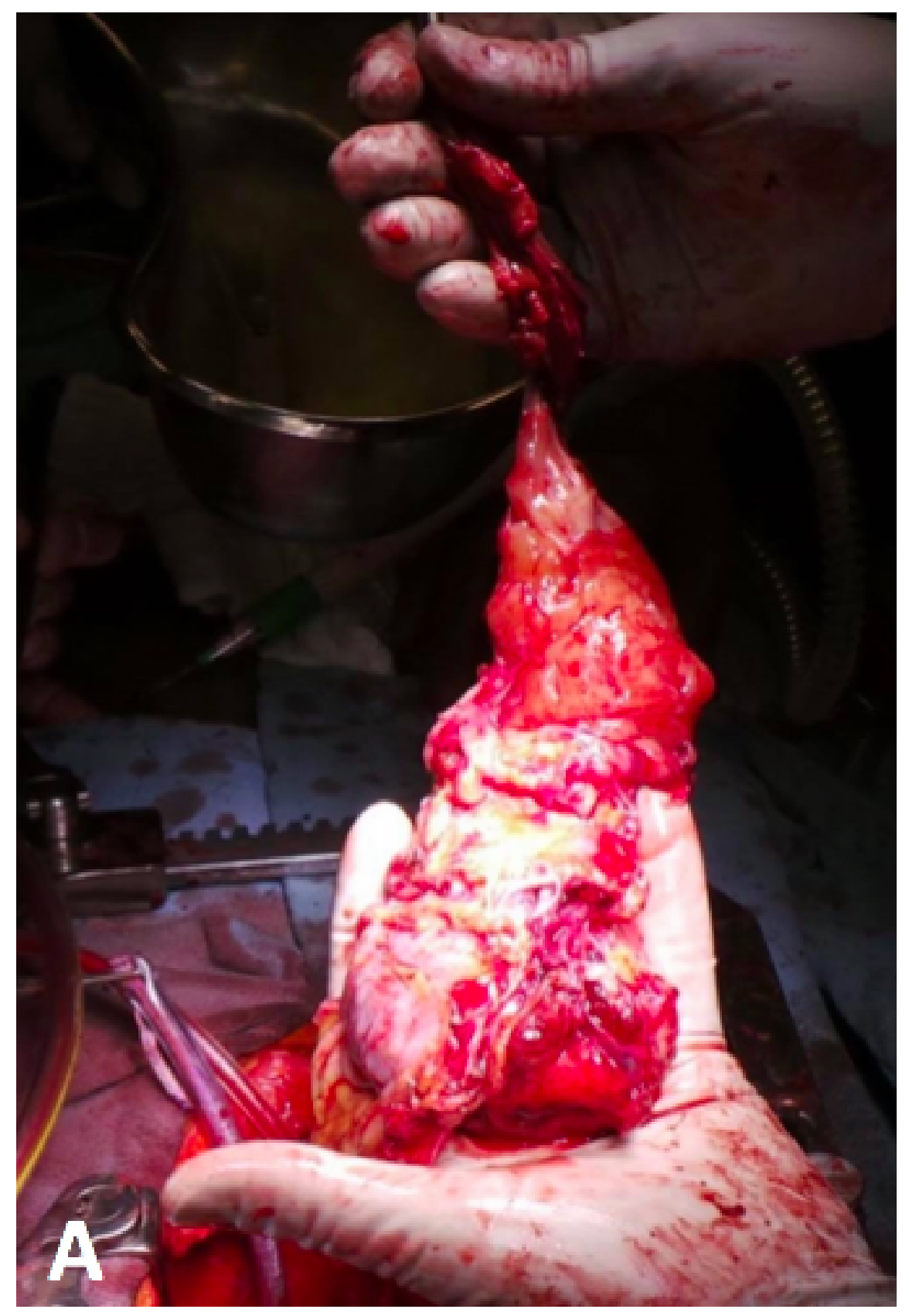




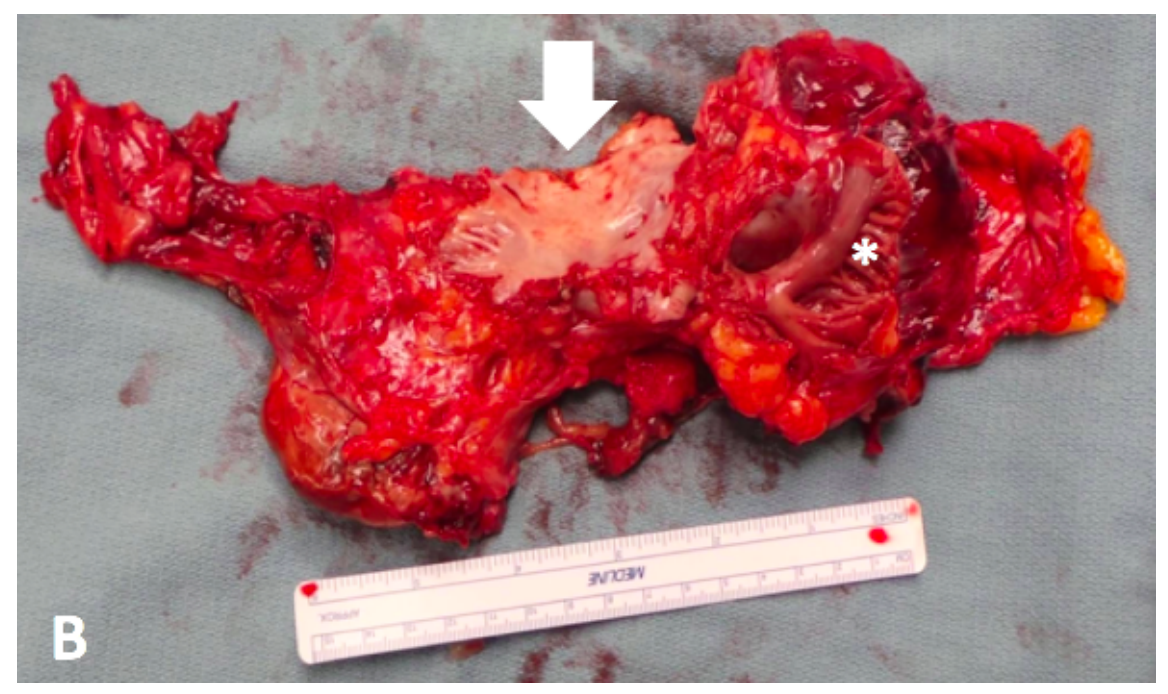

FIGURE 2. En bloc tumor resection. A and B, (A) external surface view and (B) internal surface view with superior cavoatrial junction (asterisk) and aortic root (arrow). 